\title{
On weighted three point quadrature rules
}

\author{
P. Cerone* J. Roumeliotis* G. Hanna*
}

(Received 7 August 2000)

\begin{abstract}
Weighted three point quadrature rules are obtained in the current work giving explicit a priori bounds on the error. The results are valid for general weight functions. The robustness of the bounds are explored for specific weight functions and for a variety of integrands. A comparison of the current development is made with traditional quadrature rules and it is demonstrated that the current development

* School of Communications and Informatics, Victoria University of Technology, PO Box 14428, MCMC, Victoria 8001, Australia.

mailto:Pietro.Cerone@vu.edu.au, mailto: John.Roumeliotis@vu.edu.au and mailto:georgey@matilda.vu.edu.au respectively.

${ }^{0}$ See http: //anziamj . austms .org. au/V42/CTAC99/Cero for this article and ancillary services, (c) Austral. Mathematical Soc. 2000. Published 27 Nov 2000.
\end{abstract}


has some advantages. In particular, this method allows the nodes and weights of an $n$ point rule to be easily obtained, which may be preferential if the region of integration varies. Other explicit error bounds may be obtained in advance, thus making it possible to determine the partition required to achieve a certain error tolerance.

\section{Contents}

1 Introduction

C342

2 Weighted Three Point Inequalities

C343

3 Development of a Quadrature Rule

C352

4 Numerical Results

C356

5 Concluding Remarks

C360

References

C360 


\section{Introduction}

Weighted three point quadrature rules are investigated in this article in which sampling occurs at the boundary points and an interior point. Explicit $a$ priori bounds are obtained, thus enabling the determination of the partition required for a prescribed error bound to be fulfilled. This approach contrasts to that commonly used of mesh refinement followed by a successive $a$ posteriori comparison of the results (see for example Atkinson [1]). Other quadrature rules have been developed that differ from those given here. The work in [5] presents optimal rules for given information in terms of functions in $L_{2}$. For further and more recent work see [8].

Three point quadrature rules of Newton-Cotes type have been examined in Cerone and Dragomir [2] in which the error involved the behaviour of, at most, a first derivative. Riemann and Riemann-Stieltjes integrals were examined.

In the current article, weighted three point rules are investigated in which the error relies on the behaviour of the first derivative. Roumeliotis et al. [6] investigated weighted interior point rules for twice differentiable functions.

After developing the results in the initial sections of the paper, composite quadrature rules are implemented in Section 4 and results for a log weight function are given and compared with a product-trapezoidal rule of Atkinson [1]. 


\section{Weighted Three Point Inequalities}

Weighted (or product) inequalities are to be developed. The weight function is assumed to be non-negative and integrable over its entire domain. In order to simplify the working, some notation needs to be presented.

Definition 1 Let $w:(a, b) \rightarrow[0, \infty)$ be an integrable function so that $\int_{a}^{b} w(t) d t<\infty$. Define the zeroth and first moments of $w(\cdot)$ by

$$
m(a, b)=\int_{a}^{b} w(t) d t \quad \text { and } \quad M(a, b)=\int_{a}^{b} t w(t) d t,
$$

respectively. Both are assumed to exist over the entire domain of $w(\cdot)$. The weight function may be zero at the end points.

Theorem 2 Let $f:[a, b] \rightarrow \mathrm{R}$ be a differentiable mapping on $(a, b)$ whose derivative is bounded on $(a, b)$ and denote $\left\|f^{\prime}\right\|_{\infty}=\sup _{t \in(a, b)}\left|f^{\prime}(t)\right|<\infty$. Further, let a non-negative weight function $w(\cdot)$ have the properties as outlined in Definition 1. Then for $x \in[a, b], \alpha \in[a, x], \beta \in(x, b]$, the following inequality holds

$$
\begin{array}{r}
\left|\int_{a}^{b} w(t) f(t) d t-[m(\alpha, \beta) f(x)+m(a, \alpha) f(a)+m(\beta, b) f(b)]\right| \\
\leq I(\alpha, x, \beta)\left\|f^{\prime}\right\|_{\infty},
\end{array}
$$


where

$$
I(\alpha, x, \beta)=\int_{a}^{b} k(x, t) w(t) d t \quad \text { and } \quad k(x, t)= \begin{cases}t-a, & t \in[a, \alpha] \\ |x-t|, & t \in(\alpha, \beta] \\ b-t, & t \in(\beta, b]\end{cases}
$$

Proof: Define the mapping $K(\cdot, \cdot):[a, b]^{2} \rightarrow \mathrm{R}$ by

$$
K(x, t)=\left\{\begin{array}{ll}
m(\alpha, t), & t \in[a, x] \\
m(\beta, t), & t \in(x, b]
\end{array},\right.
$$

where $m(a, b)$ is the zeroth moment of $w(\cdot)$ over the interval $[a, b]$ and is given by $(1)_{1}$.

It should be noted that $m(c, d)$ will be non-negative for $d \geq c$.

Integration by parts gives, on using (4),

$$
\begin{aligned}
& \int_{a}^{b} K(x, t) f^{\prime}(t) d t \\
= & \int_{a}^{x} m(\alpha, t) f^{\prime}(t) d t+\int_{x}^{b} m(\beta, t) f^{\prime}(t) d t \\
= & \left.m(\alpha, t) f(t)]_{t=a}^{x}+m(\beta, t) f(t)\right]_{t=x}^{b}-\int_{a}^{b} w(t) f(t) d t,
\end{aligned}
$$


producing the identity

$$
\begin{aligned}
& \int_{a}^{b} K(x, t) f^{\prime}(t) d t \\
& \quad=m(\alpha, \beta) f(x)+m(a, \alpha) f(a)+m(\beta, b) f(b)-\int_{a}^{b} w(t) f(t) d t,
\end{aligned}
$$

valid for all $x \in[a, b]$.

Taking the modulus of (5) gives

$$
\begin{aligned}
& \mid \int_{a}^{b} w(t) f(t) d t-[m(\alpha, \beta) f(x)+m(a, \alpha) f(a)+m(\beta, b) f(b)] \mid \\
&=\left|\int_{a}^{b} K(x, t) f^{\prime}(t) d t\right| \leq\left\|f^{\prime}\right\|_{\infty} \int_{a}^{b}|K(x, t)| d t .
\end{aligned}
$$

Now, we wish to determine $\int_{a}^{b}|K(x, t)| d t$. To this end notice that, from (4), $K(x, t)$ is a monotonically non-decreasing function of $t$ over each of its branches. Thus, there are points $\alpha \in[a, x]$ and $\beta \in[x, b]$ such that $K(x, \alpha)=$ $K(x, \beta)=0$.

Thus,

$$
\begin{aligned}
\int_{a}^{b}|K(x, t)| d t= & -\int_{a}^{\alpha} m(\alpha, t) d t+\int_{\alpha}^{x} m(\alpha, t) d t \\
& -\int_{x}^{\beta} m(\beta, t) d t+\int_{\beta}^{b} m(\beta, t) d t
\end{aligned}
$$


Integration by parts gives, for example,

$$
\begin{aligned}
-\int_{a}^{\alpha} m(\alpha, t) d t & =-(t-a) m(\alpha, t)]_{t=a}^{\alpha}+\int_{a}^{\alpha}(t-a) w(t) d t \\
& =\int_{a}^{\alpha}(t-a) w(t) d t .
\end{aligned}
$$

A similar development for the remainder of the three integrals on the right hand side of (7) produces the result

$$
\int_{a}^{b}|K(x, t)| d t=I(\alpha, x, \beta),
$$

where $I(\alpha, x, \beta)$ is as given by (3). Combining (6) and (8) produces the result (2) and hence the theorem is proved.

It should be noted at this stage that taking $w(\cdot) \equiv 1$ reproduces the results of Cerone and Dragomir [2]. If $\alpha=a$ and $\beta=b$ then a weighted interior point rule is obtained. If $\alpha=\beta=x$, then a weighted rule results where the function is evaluated at the boundary points. For $\alpha=a$ or $\beta=b$ then Radau type rules are obtained while the current work will focus on Lobatto type rules allowing sampling at both ends of the boundary.

Corollary 3 Inequality (2) is minimized at $x=x^{*}$ where $x^{*}$ satisfies

$$
m\left(\alpha^{*}, x^{*}\right)=m\left(x^{*}, \beta^{*}\right), \quad \alpha^{*}=\frac{a+x^{*}}{2} \quad \text { and } \quad \beta^{*}=\frac{x^{*}+b}{2} .
$$


Proof: From $(2-3), I(\alpha, x, \beta)$ may be written as

$$
\begin{aligned}
I(\alpha, x, \beta)=\int_{a}^{\alpha}(t-a) w(t) d t & +\int_{\alpha}^{x}(x-t) w(t) d t \\
& +\int_{x}^{\beta}(t-x) w(t) d t+\int_{\beta}^{b}(b-t) w(t) d t,
\end{aligned}
$$

where $\alpha \in[a, x]$ and $\beta \in(x, b]$. Equation (10) could equivalently be written in terms of its zeroth and first moments as given by (1). Differentiating (10) with respect to $\alpha, \beta$ and $x$ gives

$$
\begin{aligned}
\frac{\partial I}{\partial \alpha} & =A(\alpha, x) w(x), \quad \frac{\partial I}{\partial \beta}=B(\beta, x) w(x) \\
\text { and } \quad \frac{\partial I}{\partial x} & =m(\alpha, x)-m(x, \beta),
\end{aligned}
$$

where

$$
A(\alpha, x)=2 \alpha-(a+x), \quad B(\beta, x)=2 \beta-(x+b)
$$

and $m(\cdot, \cdot)$ is defined by $(1)_{1}$. An inspection of the second derivatives demonstrates that $(10)$ is convex on using the fact that $w(t)$ is non-negative for $t \in(a, b)$. Thus, $I$ is minimal at the zeros of (11) and so the corollary is proven.

Corollary 3 investigates the problem of determining the optimal choice of $\alpha, x$ and $\beta$ that produce the tightest bound. The following corollary gives coarser bounds although the bound may be easier to implement. 
Corollary 4 Let the conditions be as in Theorem 2. Then the following inequalities hold

$$
\begin{array}{r}
\left|\int_{a}^{b} w(t) f(t) d t-[m(\alpha, \beta) f(x)+m(a, \alpha) f(a)+m(\beta, b) f(b)]\right| \\
\leq\left\|f^{\prime}\right\|_{\infty} \times\left\{\begin{array}{c}
\|w\|_{\infty} \cdot K_{1}(x) \\
\|w\|_{1} \cdot K_{\infty}(x)
\end{array}\right.
\end{array}
$$

where

$$
K_{1}(x)=\frac{1}{2}\left[\left(\frac{b-a}{2}\right)^{2}+\left(x-\frac{a+b}{2}\right)^{2}\right]+\left(\alpha-\frac{a+x}{2}\right)^{2}+\left(\beta-\frac{x+b}{2}\right)^{2}
$$

and

$$
\begin{aligned}
K_{\infty}(x)=\frac{1}{2}\left[\frac{b-a}{2}+\mid \alpha\right. & -\frac{a+x}{2}|+| \beta-\frac{x+b}{2} \mid \\
& \left.+\left|x-\frac{a+b}{2}+\right| \alpha-\frac{a+x}{2}|-| \beta-\frac{x+b}{2}||\right]
\end{aligned}
$$

with $\|g\|_{1}:=\int_{a}^{b}|g(s)|$ ds meaning $g \in L_{1}[a, b]$, the linear space of absolutely integrable functions and $\|g\|_{\infty}:=\sup _{t \in[a, b]}|g(t)|<\infty$. 
Proof: From Theorem 2 and equations (2-3), (4) and (8) we have

$$
I(\alpha, x, \beta)=\int_{a}^{b}|K(x, t)| w(t) d t=\int_{a}^{b} k(x, t) w(t) d t .
$$

Now,

$$
\int_{a}^{b} k(x, t) w(t) d t \leq\left\{\begin{array}{l}
\|w\|_{\infty} \int_{a}^{b}|k(x, t)| d t \\
\|w\|_{1} \sup _{t \in[a, b]}|k(x, t)|
\end{array}\right.
$$

where $k(x, t)$ is as defined in $(3)$.

Some straight forward evaluation gives

$$
\int_{a}^{b}|k(x, t)| d t=\frac{1}{2}\left[(\alpha-a)^{2}+(x-\alpha)^{2}+(\beta-x)^{2}+(b-\beta)^{2}\right],
$$

which may readily be shown to equal $K_{1}(x)$ as given by (14) through using the identity

$$
\frac{X^{2}+Y^{2}}{2}=\left(\frac{X+Y}{2}\right)^{2}+\left(\frac{X-Y}{2}\right)^{2}
$$

three times.

Further,

$$
\sup _{t \in[a, b]}|k(x, t)|=\max \{\alpha-a, x-\alpha, \beta-x, b-\beta\},
$$


which can be shown to equal $K_{\infty}(x)$ as given by (15) from using the result

$$
\max \{X, Y\}=\frac{X+Y}{2}+\frac{|X-Y|}{2},
$$

three times.

Hence the corollary is proven.

It should be noted that the tightest bounds are obtained at $x=\frac{a+b}{2}$ and $\alpha=\frac{a+x}{2}, \beta=\frac{x+b}{2}$. That is, at their respective midpoints. The optimal sampling scheme is independent of the weight.

Theorem 5 Let $f: I \subseteq \mathrm{R} \rightarrow \mathrm{R}$ be a differentiable mapping on $\stackrel{\circ}{I}$ (the interior of I) and $a, b \in I$ are such that $b>a$. If $f^{\prime} \in L_{1}[a, b]$, then $\left\|f^{\prime}\right\|_{1}=\int_{a}^{b}\left|f^{\prime}(t)\right| d t<\infty$. In addition, let a non-negative weight function $w(\cdot)$ have the properties as outlined in Definition 1. Then for $x \in[a, b]$, $\alpha \in[a, x]$ and $\beta \in(x, b]$ the following inequality holds.

$$
\begin{array}{r}
\left|\int_{a}^{b} w(t) f(t) d t-[m(\alpha, \beta) f(x)+m(a, \alpha) f(a)+m(\beta, b) f(b)]\right| \\
\leq \theta(\alpha, x, \beta)\left\|f^{\prime}\right\|_{1},
\end{array}
$$

where

$$
\begin{aligned}
& \theta(\alpha, x, \beta)=\frac{1}{4}\{m(a, b)+|m(\alpha, x)-m(a, \alpha)|+|m(\beta, b)-m(x, \beta)| \\
& +|m(a, x)-m(x, b)+| m(\alpha, x)-m(a, \alpha)|-| m(\beta, b)-m(x, \beta)||\}
\end{aligned}
$$


and $m(a, b)$ is the zeroth moment of $w(\cdot)$ over $[a, b]$ as defined by $(1)_{1}$.

Proof: From identity (5) we obtain, from taking the modulus

$$
\theta(\alpha, x, \beta)=\sup _{t \in[a, b]}|K(x, t)|,
$$

where $K(x, t)$ is as given by (4). As discussed in the proof of Theorem 2, $K(x, t)$ is a monotonic non-decreasing function of $t$ in each of its two branches so that

$$
\theta(\alpha, x, \beta)=\max \{m(a, \alpha), m(\alpha, x), m(x, \beta), m(\beta, b)\} .
$$

Now, using equation (16) we have

$$
\begin{aligned}
& \qquad m_{1}=\max \{m(a, \alpha), m(\alpha, x)\}=\frac{1}{2}[m(a, x)+|m(\alpha, x)-m(a, \alpha)|] \\
& \text { and } m_{2}=\max \{m(x, \beta), m(\beta, b)\}=\frac{1}{2}[m(x, b)+|m(\beta, b)-m(x, \beta)|] \\
& \text { to give } \\
& \qquad \theta(\alpha, x, \beta)=\max \left\{m_{1}, m_{2}\right\}=\frac{m_{1}+m_{2}}{2}+\left|\frac{m_{1}-m_{2}}{2}\right|
\end{aligned}
$$

and hence the result (18) is obtained after some simplification and the theorem is proved. 
Remark 6 It should be noted that the tightest bound in (18) is obtained when $\alpha, x$ and $\beta$ are taken as their respective medians. Thus, the best quadrature rule in the above sense is given by

$$
\begin{array}{r}
\mid \int_{a}^{b} w(t) f(t) d t-[m(a, \tilde{\alpha}) f(a)+m(\tilde{\alpha}, \tilde{\beta}) f(\tilde{x}) \\
+m(\tilde{\beta}, b) f(b)] \mid \\
\leq \frac{m(a, b)}{4}\left\|f^{\prime}\right\|_{1},
\end{array}
$$

where

$$
m(a, \tilde{x})=m(\tilde{x}, b), m(a, \tilde{\alpha})=m(\tilde{\alpha}, \tilde{x}) \text { and } m(\tilde{\beta}, b)=m(\tilde{x}, \tilde{\beta}) .
$$

\section{Development of a Quadrature Rule}

The following theorem will be useful in determining the partition for composite quadrature rules. The optimal partition in terms of the partition that provides the tightest bounds will be determined. The optimal quadrature rules will result for $f^{\prime} \in L_{\infty}[a, b]$. If $f^{\prime} \in L_{1}[a, b]$ a similar development may be followed but will not be pursued further here.

Theorem 7 Let the conditions of Theorem 2 hold and let $\xi$ partition the 
interval $[a, b]$ into two. Then the following inequality holds

$$
\begin{array}{r}
\mid \int_{a}^{b} w(t) f(t) d t-\left[m\left(a, \alpha_{1}\right) f(a)+m\left(\alpha_{1}, \beta_{1}\right) f\left(x_{1}\right)+m\left(\beta_{1}, \alpha_{2}\right) f(\xi)\right. \\
\left.+m\left(\alpha_{2}, \beta_{2}\right) f\left(x_{2}\right)+m\left(\beta_{2}, b\right) f(b)\right] \mid \leq J(\mathbf{z}, \xi)\left\|f^{\prime}\right\|_{\infty}
\end{array}
$$

where

$$
J(\mathbf{z}, \xi)=J_{1}\left(\mathbf{z}_{1}, \xi\right)+J_{2}\left(\mathbf{z}_{2}, \xi\right)
$$

with

$$
\begin{aligned}
\mathbf{z}_{i}^{T} & =\left(\alpha_{i}, x_{i}, \beta_{i}\right), \quad i=1,2, \quad \mathbf{z}=\mathbf{z}_{1} \cup \mathbf{z}_{2} \\
J_{1}\left(\mathbf{z}_{1}, \xi\right) & =\int_{a}^{\xi} k_{1}\left(x_{1}, t\right) w(t) d t, \quad J_{2}\left(\mathbf{z}_{2}, \xi\right)=\int_{\xi}^{b} k_{2}\left(x_{2}, t\right) w(t) d t
\end{aligned}
$$

and

$$
\begin{aligned}
& k_{1}\left(x_{1}, t\right)= \begin{cases}t-a, & t \in\left[a, \alpha_{1}\right] \\
\left|x_{1}-t\right|, & t \in\left(\alpha_{1}, \beta_{1}\right], \\
\xi-t, & t \in\left(\beta_{1}, \xi\right]\end{cases} \\
& k_{2}\left(x_{2}, t\right)= \begin{cases}t-\xi, & t \in\left[\xi, \alpha_{2}\right] \\
\left|x_{2}-t\right|, & t \in\left(\alpha_{2}, \beta_{2}\right] . \\
b-t, & t \in\left(\beta_{2}, b\right]\end{cases}
\end{aligned}
$$

Further, $a \leq \alpha_{1} \leq x_{1} \leq \beta_{1} \leq \xi$ and $\xi \leq \alpha_{2} \leq x_{2} \leq \beta_{2} \leq b$. 
Proof: The proof follows that of Theorem 2. A subscript of 1 is used to denote parameters in the interval $[a, \xi]$ and 2 for parameters in $(\xi, b]$. Integration by parts of $\int_{a}^{\xi} K\left(x_{1}, t\right) f^{\prime}(t) d t$ produces an identity similar to (5) with $b$ replaced by $\xi$ and $x$ by $x_{1}$. Similarly for $\int_{\xi}^{b} K\left(x_{2}, t\right) f^{\prime}(t) d t$ produces an identity like (5) with $a$ replaced by $\xi$ and $x$ by $x_{2}$. Summing the two results produces an identity over $[a, b]$. Taking the modulus and using the triangle inequality, relying heavily on (3) gives the stated result after collecting the terms in order. Here on $[a, \xi],(\alpha, x, \beta, b)$ are replaced by $\left(\alpha_{1}, x_{1}, \beta_{1}, \xi\right)$ and on $[\xi, b],(a, \alpha, x, \beta)$ are replaced by $\left(\xi, \alpha_{2}, x_{2}, \beta_{2}\right)$. Hence the theorem is proved.

Corollary 8 The optimal location of the parameters in Theorem 7 are $\alpha_{1}=$ $\alpha_{1}^{*}=\frac{a+x_{1}^{*}}{2}, \beta_{1}=\beta_{1}^{*}=\frac{x_{1}^{*}+\xi^{*}}{2}, \alpha_{2}=\alpha_{2}^{*}=\frac{\xi^{*}+x_{2}^{*}}{2}, \beta_{2}=\beta_{2}^{*}=\frac{x_{2}^{*}+b}{2}$ and $x_{1}^{*}$, $x_{2}^{*}$ and $\xi^{*}$ satisfy the following respective equations: $m\left(\alpha_{1}^{*}, x_{1}^{*}\right)=m\left(x_{1}^{*}, \beta_{1}^{*}\right)$, $m\left(\alpha_{2}^{*}, x_{2}^{*}\right)=m\left(x_{2}^{*}, \beta_{2}^{*}\right)$ and $m\left(\beta_{1}^{*}, \xi^{*}\right)=m\left(\xi^{*}, \alpha_{2}^{*}\right)$.

Proof: The proof of this corollary closely follows the proof of Corollary 3. From (21-23), differentiation of $J$ with respect to $\left(\alpha_{1}, x_{1}, \beta_{1}, \xi, \alpha_{2}, x_{2}, \beta_{2}\right)$ produces, on equating to zero, seven simultaneous equations.

Using the fact that the weight function is assumed to be positive, then the solution of the seven simultaneous equations give the point at which an optimal bound is produced, since an inspection of the second derivatives readily demonstrates the convexity of the function $J$. 
The results in Theorem 7 may be used to develop a composite quadrature rule. To this end, define a grid $I_{n}: a=\xi_{0}<\xi_{1}<\cdots<\xi_{n-1}<\xi_{n}=b$ on the interval $[\mathrm{a}, \mathrm{b}]$, with $x_{i} \in\left[\xi_{i}, \xi_{i+1}\right]$ for $i=0,1, \ldots, n-1$. The following quadrature formula for weighted integrals is obtained which relies only on the first two moments of the weight function.

Theorem 9 Let the conditions in Theorem 7 hold, then following weighted quadrature rule holds

$$
\int_{a}^{b} w(t) f(t) d t=A(f, \boldsymbol{\xi}, \boldsymbol{x})+R(f, \boldsymbol{\xi}, \boldsymbol{x})
$$

where

$$
\begin{aligned}
A(f, \boldsymbol{\xi}, \boldsymbol{x})= & \sum_{i=1}^{n} m\left(\alpha_{i}, \beta_{i}\right) f\left(x_{i}\right)+m\left(\xi_{0}, \alpha_{1}\right) f\left(\xi_{0}\right) \\
& +2 \sum_{i=1}^{n-1} m\left(\beta_{i}, \xi_{i}\right) f\left(\xi_{i}\right)+m\left(\beta_{n}, \xi_{n}\right) f\left(\xi_{n}\right)
\end{aligned}
$$

and

$$
\begin{aligned}
|R(f, \boldsymbol{\xi}, \boldsymbol{x})| \leq\left\|f^{\prime}\right\|_{\infty}\left(M\left(\xi_{0}, \xi_{n}\right)-2 \sum_{i=}^{n}\right. & {\left[M\left(\alpha_{i}, \beta_{i}\right)+M\left(\beta_{i}, \xi_{i}\right)\right] } \\
+ & \left.\xi_{n} m\left(\beta_{n}, \xi_{n}\right)-\xi_{0} m\left(\xi_{0}, \alpha_{1}\right)\right) .
\end{aligned}
$$


The parameters $x_{i}, \alpha_{i}, \beta_{i}$ and $\xi_{i}$ satisfy

$$
m\left(\alpha_{i}, x_{i}\right)=m\left(x_{i}, \beta_{i}\right), \quad \alpha_{i}=\frac{\xi_{i-1}+x_{i}}{2}, \quad \beta_{i}=\frac{x_{i}+\xi_{i}}{2}
$$

for $i=1,2, \ldots, n$, and

$$
m\left(\beta_{i}, \xi_{i}\right)=m\left(\xi_{i}, \alpha_{i+1}\right),
$$

for $i=1,2, \ldots, n-1$.

Proof: Using Theorems 2 and 7 over $\left[\xi_{i}, \xi_{i+1}\right]$ for $i=0,1, \ldots, n-1$ and summing readily produces the result after using Corollaries 3 and 8 to simplify.

\section{Numerical Results}

In this section we illustrate the application of the composite quadrature rule developed in the previous section to approximate the integrals

$$
\int_{0}^{1} \frac{\ln (1 / t)}{t+2} d t=0.4484142069 \text { and } \int_{0}^{1} e^{-1 / t} \ln (1 / t) d t=0.05065230956
$$


The integrals are evaluated using the composite rule (24) and the producttrapezoidal as described in [1, p. 310]. The first integral, $(29)_{1}$, has been used to demonstrate the product-trapezoidal and as a result we can compare the performance with the rule developed here. Note that (24) is a first-order rule in that it was derived for the class of once-differentiable functions. This contrasts with the product-trapezoidal rule which is of second order. Thus, to investigate the effects of rule order, we also apply these rules to $(29)_{2}$. In contrast with $(29)_{1}$, the integrand of $(29)_{2}$ increases with the order of its derivative.

Table 1 shows the numerical error in evaluating (29) using (24) for an increasing number of intervals. We note that the nodes and weights of the quadrature rule are obtained by solving the $4 n-1$ simultaneous equations (27) and (28). It is a simple matter to implement a numerical procedure to solve these equations iteratively with an initial uniform mesh. For example on a Pentium-90 personal computer, with $n=32$, calculating (27) and (28) to 14 digit accuracy took close to 42 seconds.

Inspection of Table 1 reveals that a more accurate result is obtained for $(29)_{1}$ than for $(29)_{2}$. This is probably due to the nature of the integrands. The theoretical error ratio is consistently close to 2 . This value confirms that, due to its development, the quadrature rule is at least of first order. The numerical error ratios are somewhat larger, these values suggest 


\begin{tabular}{|c|cc|cc|c|}
\hline$n$ & \multicolumn{2}{|c|}{ Equation $(29)_{1}$} & \multicolumn{2}{|c|}{ Equation $(29)_{2}$} & \multirow{2}{*}{$\begin{array}{c}\text { Theoretical } \\
\text { Error Ratio }\end{array}$} \\
\cline { 2 - 4 } & Relative Err & Err Ratio & Relative Err & Err Ratio & \\
\hline \hline 2 & $1.64(-2)$ & & $7.27(-2)$ & & \\
4 & $4.53(-3)$ & 3.64 & $2.62(-2)$ & 2.78 & 1.70 \\
8 & $1.23(-3)$ & 3.69 & $8.47(-3)$ & 3.09 & 2.81 \\
16 & $3.29(-4)$ & 3.73 & $2.57(-3)$ & 3.30 & 2.08 \\
32 & $8.77(-5)$ & 3.75 & $7.52(-4)$ & 3.41 & 2.05 \\
64 & $2.33(-5)$ & 3.77 & $2.15(-4)$ & 3.50 & 2.03 \\
\hline
\end{tabular}

TABLE 1: The relative error in evaluating (29) using (24), where $n$ is the number of intervals.

an asymptotic form of the error bound

$$
|R(f, \boldsymbol{\xi}, \boldsymbol{x})| \sim O\left(\frac{1}{n^{\gamma}}\right), \quad \text { where } \quad \gamma \leq 2 .
$$

In Table 2 the errors in employing the product-trapezoidal rule are presented. The error ratios are consistently close to 4 which simply reflects the fact that the rule is of second order. This rule was developed by employing a linear approximation for the weighted integrand - a higher order approximation than that used here. This rule performs better than (24) for $(29)_{1}$ since the integrand is well behaved and its magnitude decreases as its derivatives increase. In contrast, the product-trapezoidal rule is inferior to (24) for $(29)_{2}$. This integrand is not well behaved and its integral is better suited to (24) 


\begin{tabular}{|c|cc|cc|}
\hline$n$ & \multicolumn{2}{|c|}{ Equation $(29)_{1}$} & \multicolumn{2}{c|}{ Equation $(29)_{2}$} \\
\cline { 2 - 5 } & Relative Error & Error Ratio & Relative Error & Error Ratio \\
\hline \hline 2 & $7.12(-3)$ & & $4.29(-1)$ & \\
4 & $1.98(-3)$ & 3.60 & $8.08(-2)$ & 5.30 \\
8 & $5.17(-4)$ & 3.83 & $1.90(-2)$ & 4.25 \\
16 & $1.32(-4)$ & 3.92 & $4.74(-3)$ & 4.01 \\
32 & $3.33(-5)$ & 3.96 & $1.18(-3)$ & 4.00 \\
64 & $8.35(-6)$ & 3.98 & $2.96(-4)$ & 4.00 \\
\hline
\end{tabular}

TABLE 2: The relative error in evaluating (29) using the product trapezoidal rule [1, p. 310], where $n$ is the number of intervals.

which was developed for a more general class of function.

We note that the product-trapezoidal rule employs a uniform mesh and the behaviour of the weight function, $w(t)$, is accounted for in the quadrature rule weight. Rules of this type were explored in [6], where a one-point, second order product rule was developed. In this paper, Roumeliotis et al., showed that, for the log weight, employing a non-uniform mesh, similar to (28) increases accuracy by a factor of more than two for $f^{\prime \prime} \in L_{\infty}[a, b]$.

Finally, we note that the rule developed here is composite in nature and identifies an "optimal" partition for an arbitrary weight. This contrasts with Gauss quadrature [7] which is not composite and hence provides no information as to how one should partition. 


\section{Concluding Remarks}

The approach described enables the user to predetermine the partition required to assure the result to be within a certain error tolerance. This approach is somewhat different from that commonly used of systematic mesh refinement followed by a comparison of successive approximations which forms the basis of a stopping rule. See $[1,3,4]$ for a comprehensive treatment of traditional methods.

Although the bounds were obtained in terms of the behaviour of the first derivative the methodology may be extended to involve higher derivatives. It may be advantageous to rely on the behaviour of lower derivatives as demonstrated in the evaluation of $(29)_{2}$ in which the higher derivatives are badly behaved.

\section{References}

[1] K. E. Atkinson. An Introduction to Numerical Analysis. John Wiley, 1989. C342, C342, C357, C359, C360

[2] P. Cerone and S. S. Dragomir. Three point quadrature rules involving, at most, a first derivative. SIAM J. Math. Anal., submitted, 1999. C342, C346 
[3] H. Engels. Numerical Quadrature and Cubature. Academic Press, New York, 1980. C360

[4] A. R. Krommer and C. W. Ueberhuber. Numerical Integration on Advanced Computer Systems. Lecture Notes in Computer Science No. 848, Berlin, 1994. Springer-Verlag. C360

[5] M. Golomb and H. F. Weinberger. Optimal approximation and error bounds. In R. E. Langer, editor, On Numerical Approximation, pages 117-190, Madison, 1959. University of Wisconsin Press. C342

[6] J. Roumeliotis, P. Cerone and S. S. Dragomir. An Ostrowski type inequality for weighted mappings with bounded second derivative. J. KSIAM, 3(2):107-119, 1999. C342, C359

[7] A. H. Stroud and D. Secrest. Gaussian quadrature formulas. Prentice Hall, 1966. C359

[8] J. F. Traub and H. Woźniakowski. A General Theory of Optimal Algorithms. Academic Press, 1980. C342 